\title{
Nursing Documentation: A survey of Hemodialysis Documentation Status at Kenyatta National Hospital's Renal Unit \\ ${ }^{1 *}$ Social Services League Hospital, Nairobi. \\ ${ }^{2}$ School of Nursing Sciences, MasindeMuliro University of Science and Technology. ppamessa@yahoo.com
}

\begin{abstract}
Background: Documentation provides communication regarding the care of clients and acts as a formal legal document giving details of a client's management. Throughout the world it is accepted that if a procedure is not documented it is not done thus documentation should be complete and accurate. Timely, accurate and complete documentation helps the patient secure better care and protects the nurse from litigation.

Objective: To assess the completeness and accuracy of nursing documentation at Kenyatta National Hospital's renal unit.

Design: Cross-sectional descriptive study.

Results:77.4\% of the charts had patient numbers indicated. All the charts did not have laboratory tests or results indicated. $95.7 \%$ of the charts did not have instructions to the patients on discharge. Less than $30 \%$ of the charts were complete and accurate.

Conclusion: Documentation was not accurate nor complete.

Recommendation: The nurses working in the renal unit should have continuous medical education on documentation and there should be audits done on documentation. Further research is also recommended on nursing documentation.
\end{abstract}

Keywords: Nursing documentation, accuracy, medical records, Kenyatta National Hospital. INTRODUCTION

Documentation is a valuable method of demonstrating that a nurse has applied nursing knowledge, skills and judgment as per the professional nursing standards. Hence, nursing documentation is a record of nursing care that is planned and delivered to individual clients by qualified nurses or other caregivers under the direction of a qualified nurse (Urquhart et al, 2009). It is also a vital tool for effective communication amongst health care team members. Nursing documentation has been one of the most important functions of nurses since the time of Florence Nightingale as it serves multiple and diverse purposes. Currently, there is an evolving quality agenda in healthcare that has significant implications for acceptable documentation requirements. This makes documentation importantin evaluating care conducted by a caregiver. Proper documentation is essential in an event of law suit as it provides evidence that the care that was provided met professional standards (Gugerty et al, 2007).

\section{NURSING DOCUMENTATION}

\section{Importance of nursing documentation}

Nursing documentation is the written evidence of nursing practice that reflects accountability of nurses to patients.Since the time of Florence Nightingale, nurses have viewed documentation as a vital part of professional www.arjonline.org 
Nursing documentation: A survey of Hemodialysis documentation status at Kenyatta National Hospital's Renal Unit

practice (Iyer, 1995).It is generally recognized across the world as one of the important duties underscoring professional autonomy and serving as the center of nursing activities (Isola et al, 2004). Documentation is reported to take up to $50 \%$ of nurses' time per shift making nurses to view it as tedious and time consuming. According to Gugerty 2007, nursing documentation serves a number of important functions including communication, continuity of care, accountability, funding, resource allocation, expanding the science of nursing and for legal purposes. It can also be used to predict mortality and serves as an indicator of quality nursing practice on the care that the nurses provide (DeLaune\& Ladner, 2011).

Accurate, detailed documentation shows the extent and quality of care that nurses provide, outcomes of the care provided,treatment and education given to the clients. Thorough, accurate documentation is important for continuity of care and decreases the potential for miscommunication and errors.

Proper documentation demonstrates a nurse's knowledge and judgment skills. The importance of proper documentation in nursing cannot be overrated since failure to document can result in poor outcomes for patients and liability issues (Urquhart et al, 2009).It is essential for nursing practice as it reflects the nursing process because it is an important aspect of every nurse's job, as the old saying goes "if it is not documented it is not done". What is documented provides evidence of what has been done and provides evidence of care that has been provided (Wang, 2011).

\section{Quality of nursing documentation}

Nursing documentation must provide an accurate, complete and honest account of the events that occurred and when these events occurredin every practice setting.Accurate nursing documentation enables nurses to systematically review the nursing process and evaluate the quality of care. Documentation should be in a chronological order with indication of late entry as defined by the institutional policies. Documentation should be done at the next available entry space and empty lines should not be left for another person to add documentation. In case there is an empty line, the nurse should draw a line from the end of the entry to the signature (Wang, 2011).

Assessment of nurses' reports in patient records can be helpful in improving the accuracy of nursing documentation. Nurses are on the front lines of patient care hence, their written accounts are critical for planning and evaluation of medical interventions and ongoing patient.Nurses are responsible for maintaining accurate records of the care they providecare (Austin, 2011; Potter \& Perry, 2010).

The quality of nursing documentation is a multidimensional concept in the form of structure, format, process and content. Structure and format relate to constructive features and physical presentation of records such as quantity, completeness, legibility and use of abbreviations that are standard and universal.Nurses should also provide a full signature or initials and professional designation with all documentation. The hand-written documentation should be legible and complete with permanent ink. The documentation content should relate to the message on the care process provided. It is concerned with comprehensiveness, appropriateness and the relationship of the steps of nursing process (Wang et al, 2011).A study by the National Client Safety Agency (NPSA) found that poor standards of documentation were a contributory factor in failure to detecting clients who were in clinically deterioratingstates (NPSA, 2007).

\section{Purposes of nursing documentation}

\section{Communication}

Nursing documentation is essential for good communication within the multidisciplinary team on the patient's progress and general condition.Nurses should ensure that documentation presents an accurate, clear and comprehensive picture of the client's needs, nurse's interventions and client's outcomes (Juan and Moyet, 2004). It plays a significant role in the delivery of nursing care services through supporting better communication 
Nursing documentation: A survey of Hemodialysis documentation status at Kenyatta National Hospital's Renal Unit

between different care team members to facilitate continuity of care and safety of the clients. Inaccurate or incomplete documentation leads to care that is fragmented, tasks that are repeated and therapies which could be delayed or omitted due to a break in the chain of communication (Perry \& Potter, 2012).According to the Joint Commission 2012, poor communication contributes significantly to the occurrence of adverse events in healthcare making it an important target of initiatives to improve patient safety. Patient safety is of utmost importance to the healthcare institution and should always be ensured (JCIA, 2012).

\section{Accountability}

Documentation is a valuable method of demonstrating that nursing knowledge, judgment and skills have been applied within a nurse-client relationship in accordance with the standards of practice. Nurses are expected to follow the standards of practice and code of ethics when documenting information related to the health status of clients, circumstances and care provided. Nurses are accountable to the care that they give the patients, this can only be proved through documentation. Through chart audits and performance reviews, documentation is used to evaluate the quality of services and appropriateness of care provided (NPSA, 2007; Wang et al, 2011).

\section{Legal purposes}

The client's record is a legal document that can be used as evidence in a court of law or in a professional conduct proceeding. Documentation provides evidence of care and is a medico legal requirement for nursing practice. Documentation should provide a chronological record of events in client care and delivery of services. Courts may use the health record to reconstruct events, establish time and dates, and refresh one's memory and to substantiate and/or resolve conflicts in testimony. In a court of law, the patient's health record serves as the legal record of the care provided to that patient. Hence, it is important that nurses document all the care they provide to the patients in a chronological order and according to the hospital policies (CNPS, 2009; Austin, 2011).

\section{Documentation errors}

While documenting, each sheet of the medical record should be correctly labeled with the patient's identification information indicated to avoid documenting on the wrong patient or mistaking the patient for another. If a patient sustains an injury, it could be determined that there was neglect based on lack of documentation. Most times, nursing records have no nursing diagnoses or if nursing diagnoses are identified the patients' problems that are identified predominantly address physical problems based on medical diagnoses with few psychological needs. Nursing interventions recorded are related to medical rather than nursing problems and rarely include the patients' view. All these often show legal inaccuracies. The Plaintiff's attorney looks for facts to prove each of the elements that can lead to a law suit when reviewing medical records. Hence, it's important to follow guidelines to prevent a plaintiff's attorney from raising questions about the quality of care a patient was given (Austin, 2011).

Numerous documentation forms produce data redundancy, inconsistency and irregularity of charting. It's important to know the medications that patients are taking and allergies. While documenting, nurses should indicate each medication administered, dosages and patient reactions.Blank spaces on a chart or document do more than fail to provide necessary information and also create ambiguity. A patient who sues has a much stronger case if treatment was not documented even if it was provided, there is no way to prove the treatment occurred.Every single entry should have the date, time and name of the person who entered it. All entries should be signed using the first and last name and title of the nurse to clearly identify who wrote the entry. The nurse should sign his/her initials on the document using full signature in the appropriate place for identity as the care provider. When documentation continues from one page to the next, the name of the nurse should be written on each page along with the date and time on all subsequent pages (Perry \& Potter, 2010). 
Nursing documentation: A survey of Hemodialysis documentation status at Kenyatta National Hospital's Renal Unit

Writing illegibly can result in confusion and communication problems that can lead to inefficiencies costing patients their lives. Illegible writing can also interfere with defense in a malpractice suit.Misspelled words and/ or illegible entries can result in misinterpretation of information and could result in client harm. Correct spelling and legibility of nursing documentation demonstrates a level of competency and attention to detail.If an error is made, a single line should be drawn through the mistake and a notation made such as "documentation error"(Perry \& Potter, 2010).A number of frameworks are currently available to assist with nursing documentation including narrative charting, problem orientated approaches, clinical pathways, and focus notes. However many nurses still experience barriers to maintaining accurate and legally prudent documentation.

\section{Conclusion}

Quality documentation is an integral part of professional nursing practice. It reflects the application of nursing knowledge, skills and judgment. This supports the nurse's contribution to development of agency policy and promotes evidence informed practice which enables nurses to meet standards of practice for registered nurses every day in client care.

\section{Materials ANd Methods}

\section{Study design}

A cross-sectional quantitative designwas adopted to assess nursing documentation for accuracy, completeness and continuity of patients undergoing hemodialysis. The study was carried out inthe months of March to April 2015.

\section{Study area}

Kenyatta National Hospital's (KNH) Renal Unit which currently has a bed capacity is 25, admitting patients undergoinghemodialysis.

\section{Sampling technique}

Systematic random sampling was employed and 180 nursing charts for patients undergoing hemodialysis was used.

\section{Data collection procedure and tools}

A checklist was designed and used to collect data. The study also incorporated D-Catch instrument to assess accuracy of documentation. Analysis of data was done using statistical packages of Social Sciences version 20 as well as manual calculation.

\section{Ethics}

Permission to access nurses' records was obtained from the nurse in charge of the renal unit as well as the health records officer within the unit.

\section{RESULTS}

\section{Completeness of documentation}

Incompleteness in charting is a problem with the existing nursing documentation. It was noted that $77.4 \%$ of the charts had patient identification numbers with $100 \%$ having names indicated.It was also noted that $100 \%$ of the charts did not have pre and post-dialysis laboratory tests done and results documented, $93.3 \%$ did not have intra dialysis interventions, $89.6 \%$ did not discharge patients post dialysis and $95.7 \%$ did not give client instructions and education on discharge as illustrated in figure 1 below. 
Nursing documentation: A survey of Hemodialysis documentation status at Kenyatta National Hospital's Renal Unit

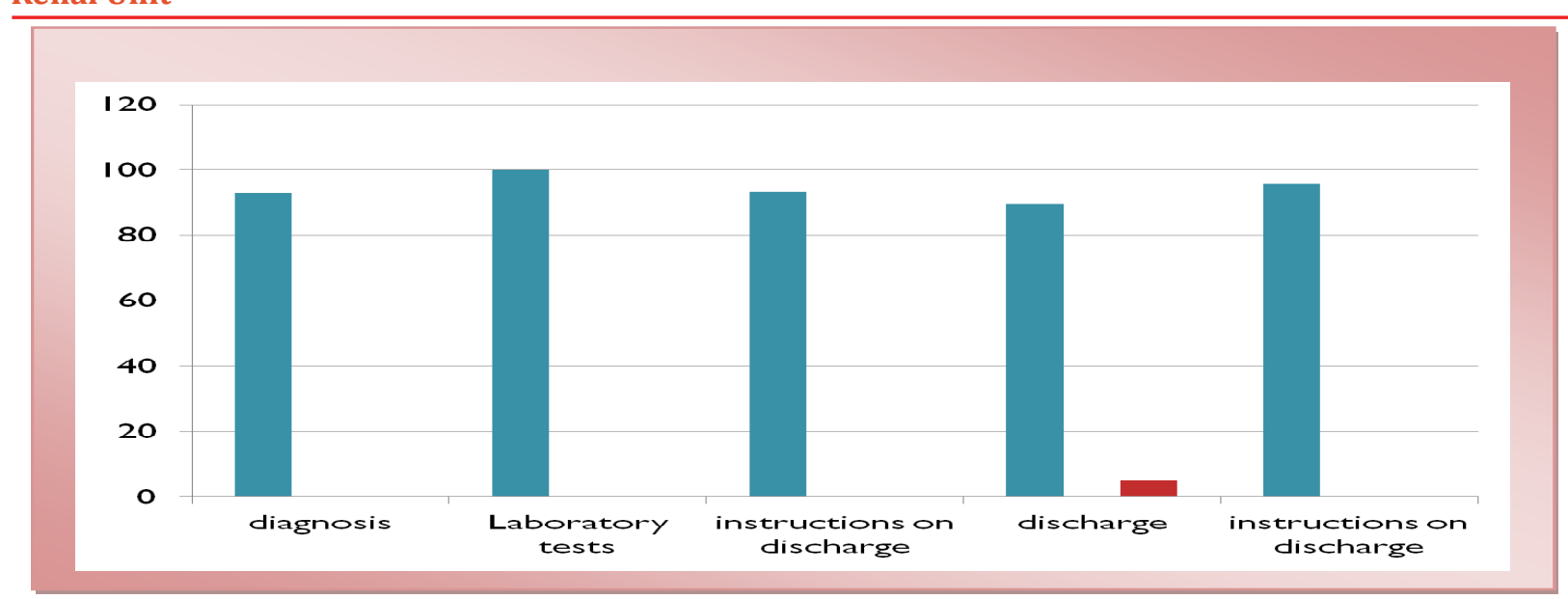

Fig1. Missing information on the charts

\section{Accuracy of documentation}

Most of the charts had inaccuracies. The nurses were keen on indicating patients' names with some patients having identical names rather than their identification numbers since $100 \%$ had patient names with only $77.4 \%$ having admission numbers and names. $88.9 \%$ had the time dialysis begun indicated, $83 \%$ had dialysis instructions, $77.6 \%$ had intra dialysis vitals indicated. Only36.6\% indicated post dialysis interventions as shown in Table 1 below. It was also noted that less than $30 \%$ of the documentation had been done accurately.

Table 1: Accurate and inaccurate documentation

\begin{tabular}{|l|l|}
\hline Accurate documentation & Inaccurate documentation \\
\hline In/outpatient numbers (77.4\%) & No pre/post dialysis laboratory tests (100\%) \\
\hline Names of client (100\%) & No intra-dialysis interventions (93.3\%) \\
\hline Time dialysis begun (88.9\%) & No discharge of clients (89.6\%) \\
\hline Intra-dialysis vital signs (77.6\%) & No post dialysis interventions (36.6\%) \\
\hline Dialysis instructions (83\%) & No discharge instructions (95.7\%) \\
\hline & No client diagnosis (93\%) \\
\hline
\end{tabular}

There were documentation errors noted related to poor cancelation, illegible documentation and poor charting as illustrated below. Poor cancelation is illustrated in Figure 2 and 3 below.

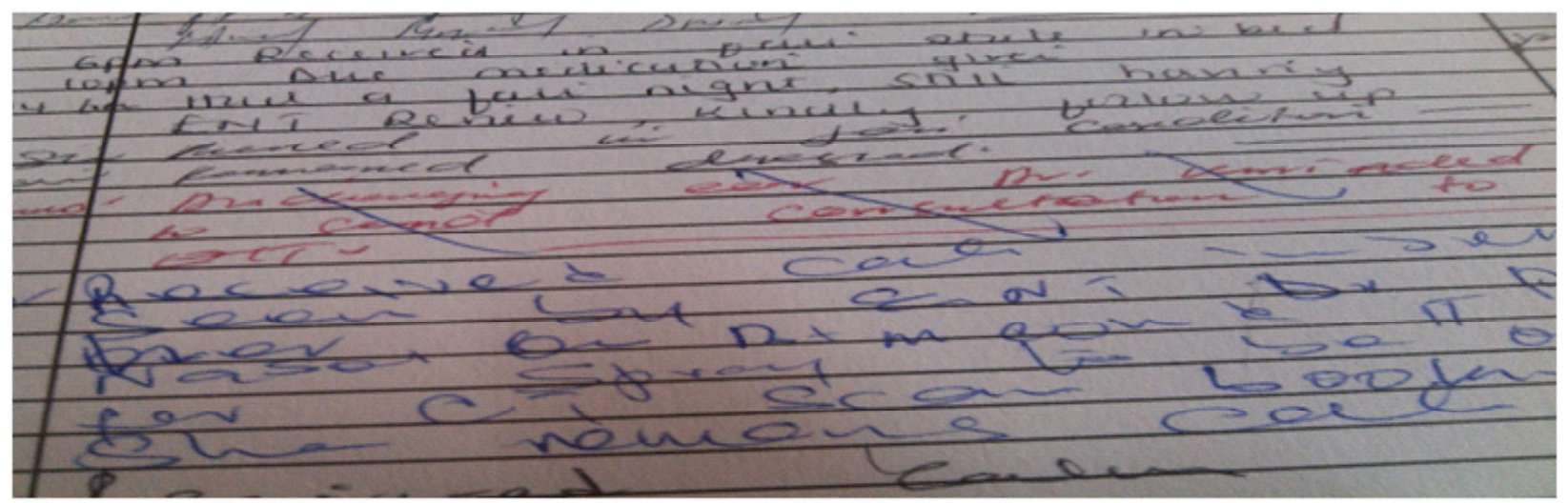

Fig2. Poor cancelation 
Nursing documentation: A survey of Hemodialysis documentation status at Kenyatta National Hospital's Renal Unit

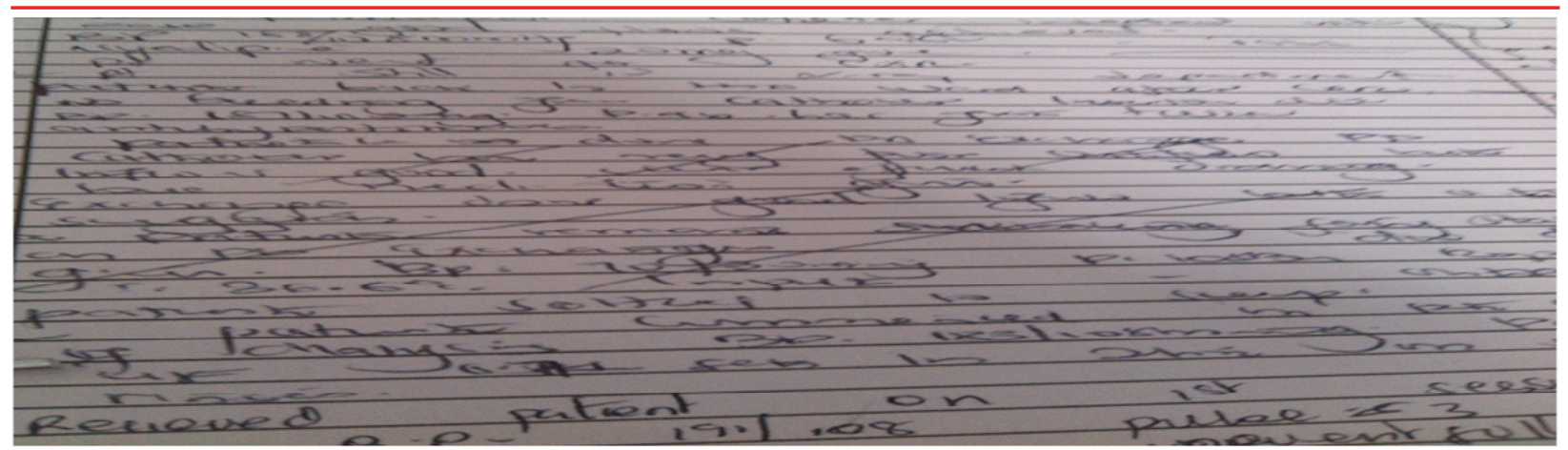

Fig3: Poor cancelation

It was also noted that most of the nurses had illegible documentation as illustrated in figure 3 below.

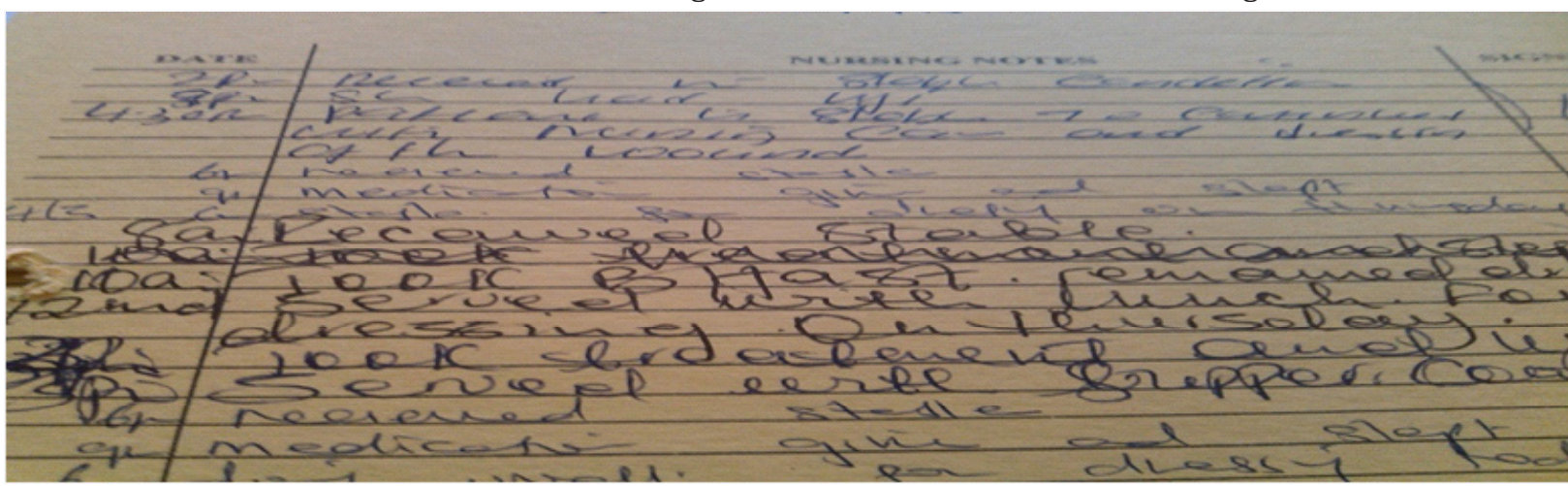

Fig3. Illegible documentation

\section{Continuity in documentation}

All the charts did not have evidence ofcontinuity of care provided. From most of the charts, only the initial vital signs were indicated without continuity in the hourly monitoring intra-dialysis at $85 \%$. It was also noted that $95 \%$ of the charts did not have post dialysis findings and interventions. Some of the vital signs documented were incomplete, it was noted that only $67.5 \%$ of the charts had weight and blood pressure documented. Figure 3 illustrates a properly filled chart while figure 4 illustrates an improperly filled chart.

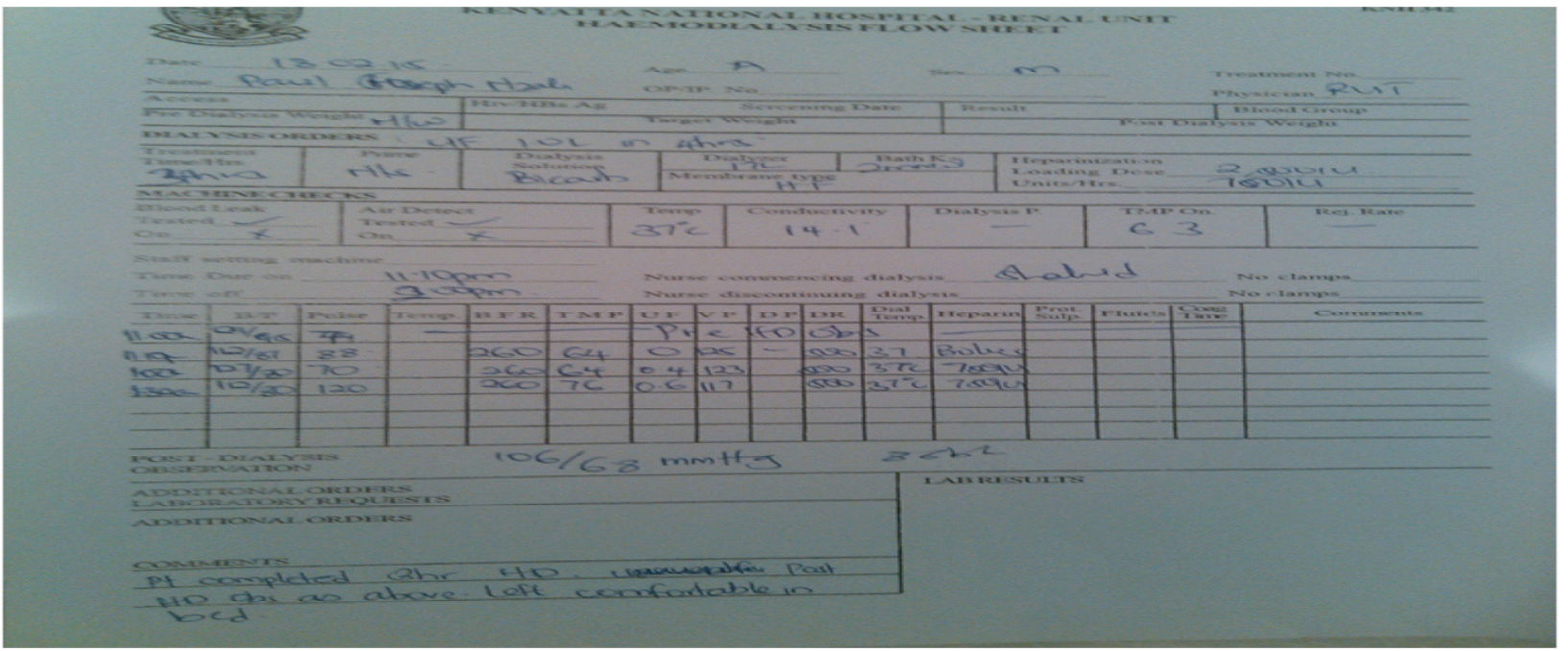

Fig4. Correctly filled chart 


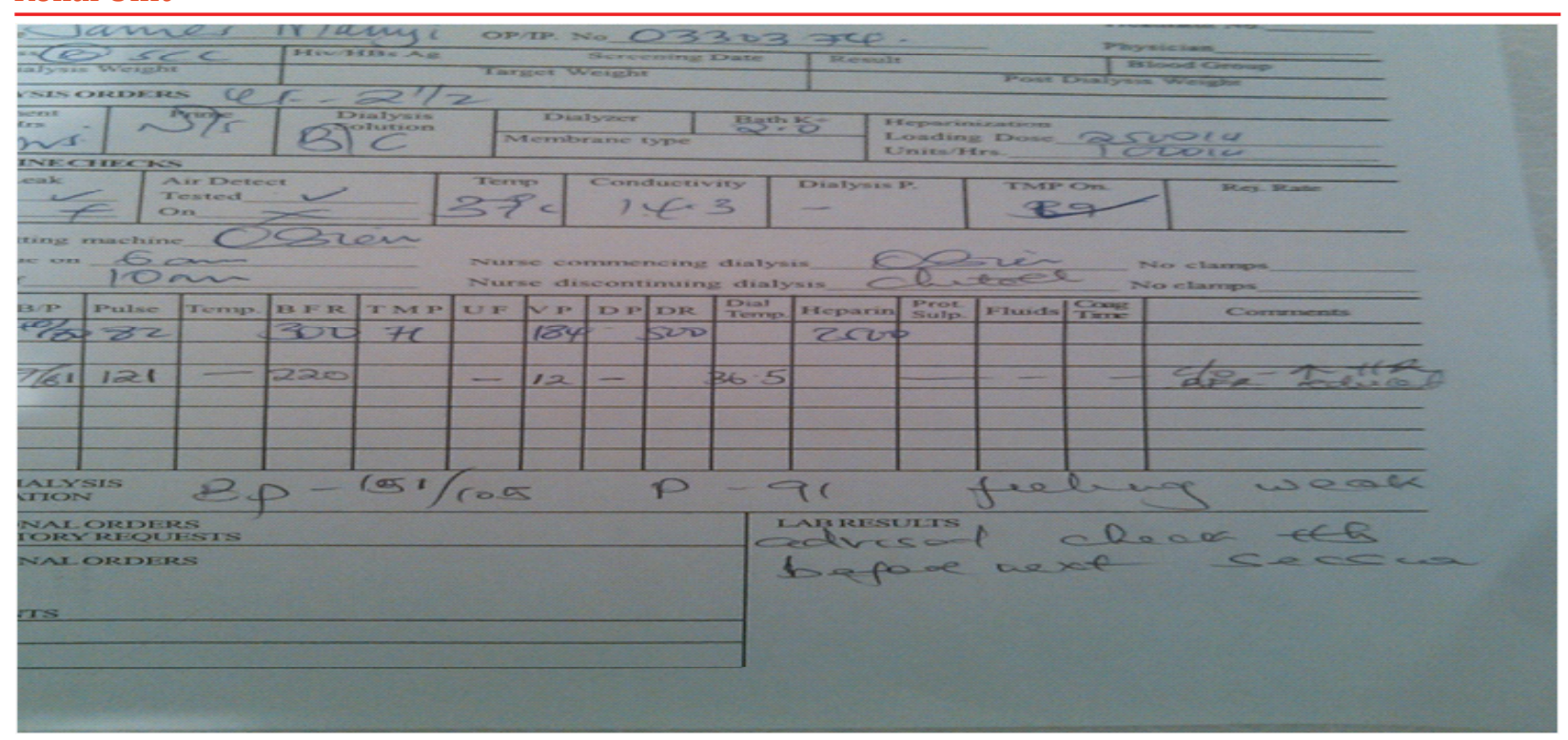

Fig5. Incorrectly filled chart

\section{DISCUSSION}

According to Prideaux (2011), the quality of nursing documentation in many countries remains poor.This was in concurrent with this study whereby most of the charts had poor documentation was noted. A Dutch study found inadequate documentation of important aspects of assessment and other related nursing care including inaccuracy in documentation (Paans et al. 2010). This is also related to the current study whereby most charts were either incomplete or lacked pertinent data that was crucial to the patient's condition pre, intra and post hemodialysis. It was noted that only $36.6 \%$ of the charts had post hemodialysis instructions indicated. Clinical records should contain sufficient information to identify the patient, support diagnosis, justify treatment and document course and results of treatment.

A Swedish study by Gunningberg\& Ehrenberg (2004) reported incongruence between what was documented and the actual physical patient status. From this survey, it was noted that only $77.6 \%$ of the charts had intra-dialysis vital signs indicated as required though there was no documentation on the nursing interventions in the events of changes in the vital signs. During dialysis the nurse should document vital signs hourly and incase of changes there should be interventions done that should be clearly documented. This is in congruent with an Australian study by Jefferies et al (2011) which had findings of limited documentation of the work of nurses.

\section{CONCLUSION}

There was poor documentation of nursing assessments and interventions for patients undergoing hemodialysis at the renal unit of Kenyatta National Hospital. Evidence of proper documentation remains a challenge in clinical practice settings worldwide. The hemodialysis charts were poorly filled with no hourly documentation of the dialysis progress as well as vital signs. Overall, there was incomplete documentation with failure to chart patients' weights and other pertinent patient data. It was noted that the patients were not being identified by the use of two identifiers. It was also noted that there was use of abbreviations that were not standard with improper cancelation of the errors which could not be used for legal purposes as the documentation may not provide prove of in a court of law. Effective documentation systems should support nurses in linking diagnoses, interventions,progress and outcome evaluations. To improve the accuracy of documentation, further research is needed on the factors that influence poor nursing documentation. 


\section{RECOMMENDATION}

Continuous Medical Education on documentation is necessary, nurses should be educated on the importance of proper documentation with emphasis on completeness and accuracy. It is also important that the nurses are informed of the importance of ensuring that there are no blank spaces as well as proper cancelation. Emphasis should be put on indicating the names and signatures against every entry made by the nurses during their documentation.

There should be audits done on either quarterly or more frequently to check and assess patients' clinical records for content and completeness. This should focus on timeliness, legibility and completeness. The results of the review process should be incorporated into the hospital's quality oversight mechanisms.

Further research is recommended so as to identify the causes of poor documentation as well as barriers to documentation from the nurses. These researches should be done in the renal units as well as other units. This would improve on documentation and ensure that it is of quality and standard as per the requirements. Workplace environment can contribute to poor documentation in that heavy workloads, laborious documentation forms, inadequate resources and hospital culture all impact the quality of nurses' documentation.

\section{ACKNOWLEDGEMENT}

The authors would like to thank and acknowledge the nurses at Kenyatta National Hospital's Renal Unit.

\section{CONFLICT OF INTEREST}

The authors declare that they have no financial or personal relationship(s) that may have inappropriately influenced them in writing this article.

\section{REFERENCES}

Austin S, Stay out of court with proper documentation, Nursing, 2011, 41 (4): 24 - 29.

Blair W \& Smith B, Nursing documentation: frameworks and Barriers, Contemporary Nurse,2012, 41 (2).

DeLaune C.S\& Ladner K.P, Fundamentals of nursing standards \& practice, 2011. $4^{\text {th }}$ Edition. Cengage Learning, New York.

Gugerty B, Maranda M.J, Beachley M, Navarro V.B, Newbold S et al, Challenges and opportunities in documentation of the nursing care of patients. Baltimore, documentation work group, Maryland Nursing Workforce commission.

Iyer P.W, Camp N.M, A nursing process approach, $2^{\text {nd } E d n . ~ S t . ~ L o u i s, ~ M O, ~ U S A: ~ M o s b y s-y e a r ~ b o o k, ~} 1995$

Jefferies D, Johnson M \& Griffiths R, A meta-study of the essentials of quality nursing documentation, International Journal of Nursing Practice, 2011, 16, 112 -124.

National Clients Safety Agency 2007. Recognizing and responding appropriately to signs of deterioration in hospital clients. NPSA London. 2007

Okaisu E.M, Kalikwani F, Wanyana G \& Coetzee M, Improving the quality of nursing documentation: An action research project, Journal of the Democratic Nursing Organisation of South Africa, 2014, 37, 2

Paans W, Sermeus W, Niewag R.M \& Van der Schans C.P, Prevalence of accurate nursing documentation in patient records, Journal of Advanced Nursing, 2010,66(11), 2481 - 2489. 
Nursing documentation: A survey of Hemodialysis documentation status at Kenyatta National Hospital's Renal Unit

Prideaux A, Issues in nursing documentation and record-keeping practice, British Journal of Nursing.2011, 20(22), $1450-1454$.

Potter P.A \& Perry A.G, Canadian fundamentals of nursing, 2010, Toronto. Elsevier.

The Joint Commission, 2012, 'Sentinel Event Data: Root causes by event type 2004 - 2012', http://www. jointcommision.org/assets/1/18/Root_Causes_Event_Type_04_4Q2012.pdf

Urquhart C, Currel R, Grant M.J, Hardiker N.R, Nursing record systems: Effects on nursing practice and healthcare outcomes, Cochrane Data Base of Systematic Reviews, 2009, 1: 1 - 66.

Wang N, Hailey D, Yu P, Quality of nursing documentation and approaches to its evaluation: A mixed method systematic review, Journal of Advanced Nursing, 2011; 67: 1858 - 1875.

Citation: Assanga Amesa Phoebe, Isiaho Amugutsi Lilian "Nursing documentation: A survey of Hemodialysis documentation status at Kenyatta National Hospital's Renal Unit". American Research Journal of Nursing. 2017; 3(1): 1-9.

Copyright (c) 2017 Assanga Amesa Phoebe, Isiaho Amugutsi Lilian, This is an open access article distributed under the Creative Commons Attribution License, which permits unrestricted use, distribution, and reproduction in any medium, provided the original work is properly cited. 